\title{
La placa grabada de Balma Guilanyà (Prepirineo de Lleida) y las manifestaciones artísticas del Mesolítico de la Península Ibérica
}

\author{
The carved rock of Balma Guilanyà (Prepyrenees of Lleida) and the Mesolithic art \\ in the Iberian Peninsula
}

Jorge Martínez-Moreno (*)

Valentín Villaverde $(* *)$

Rafael Mora Torcal $(*)$

\section{RESUMEN}

El hallazgo de un bloque grabado de grandes dimensiones en Balma Guilanyà, con motivos geométricos $\mathrm{y} / \mathrm{o}$ abstractos permite analizar la problemática referida a las manifestaciones artísticas que se desarrollan con posterioridad al arte del Paleolítico Superior en la región mediterránea ibérica. En este artículo se presenta el contexto arqueoestratigráfico, cronométrico y cronocultural de esta manifestación artística, durante el X milenio cal BP. $\mathrm{El}$ análisis de los motivos gráficos y su comparación con otras representaciones muebles y parietales de la vertiente mediterránea ibérica permite evaluar la problemática que rodea la caracterización del arte mesolítico en esta zona.

\begin{abstract}
A carved rock with geometric and/or abstract signs discovered at the Balma Guilanyà site has made possible the analysis of the artistic patterns developed after the end of the Upper Palaeolithic in the Iberian Mediterranean region. Archaeo-stratigraphic, chronometric and chrono-cultural attributes link this finding to the Mesolithic, probably during the tenth millennium cal BP. Graphic analysis and the comparison with different kinds of representations from this same area allows the eva-
\end{abstract}

(*) Centre d'Estudis del Patrimoni Arqueològic de la Prehistoria. Facultat de Lletres. Universitat Autònoma de Barcelona. 08193 Bellaterra. Correos e.: Jorge.martinez@uab.cat; Rafael.mora@uab.cat

(**) Dpt. Prehistòria i Arqueologia. Universitat de València. Blasco Ibáñez 28. 46010 Valencia. Correo e.:

Valentin.villaverde@uv.es

Recibido: 6-X-2009; aceptado: 29-I-2010. luation of the problematic that surrounds the characterization of West Mediterranean Mesolithic art.

Palabras clave: Balma Guilanyà; Arte mobiliar; Mesolítico; Mediterráneo occidental; Holoceno antiguo.

Key words: Balma Guilanyà; Portable art; Mesolithic; Western Mediterranean; Early Holocene.

\section{LAS MANIFESTACIONES ARTÍSTICAS DEL MESOLÍTICO EN LA PENÍNSULA IBÉRICA}

Las manifestaciones artísticas mesolíticas del Mediterráneo ibérico, pese no ser muy abundantes, son conocidas desde la primera mitad del siglo pasado. Aunque la posición cronológica de la plaqueta con una cierva grabada del nivel 2 de Sant Gregori (Vilaseca 1934) puede resultar incierta, la cronología holocena de las piezas de Filador (Vilaseca 1949) y Cocina (Pericot 1945) plantea escasas dudas, habida cuenta de los contextos industriales a los que se asocian.

El número de yacimientos que han proporcionado piezas de arte mueble del final del Paleolítico es más elevado, pero la incertidumbre en torno a la posición cronoestratigráfica de muchas de ellas es importante. Esta circunstancia, que impedía una pormenorizada valoración del final del ciclo artístico paleolítico, recientemente ha sido modificada tras los hallazgos efectuados en algunos yacimientos del sur de Cataluña y el norte del País Valenciano. Conjuntos como los de Molí del 
Salt (García Díez y Vaquero 2006), Parpalló (Villaverde 2004), Matutano (Olaria 2008) o Tossal de la Roca (Ripoll y Cacho 1987), parecen confirmar una marcada continuidad entre el arte del Magdaleniense superior-final y el del Epipaleolítico microlaminar no sauveterroide, englobando aquellos conjuntos líticos que por sus características tecno-tipológicas se adscriben al Epimagdaleniense (1). Las recientes síntesis que caracterizan el arte parietal en la zona septentrional valenciana, adscritas al final del ciclo artístico paleolítico (Villaverde 2004, 2005; García Díez y Vaquero 2006), inciden en la idea que estas representaciones figurativas presentan rasgos de simplificación, geometrización y estilización similares a los que aparecen en gran parte de Europa occidental en este período (Guy 1993; D’Errico y Possenti 1999; Bueno et al. 2007).

Por el contrario, las pocas novedades que en los últimos años ha ofrecido el arte mesolítico de la vertiente mediterránea ibérica han favorecido una falsa idea de continuidad entre las grafías figurativas del Epimagdaleniense y el arte mesolítico, representado por las plaquetas de Cocina (Pericot 1945; Pascual 2008). Las únicas excepciones son las piezas procedentes de Filador ( $\mathrm{Fu}-$ llola y Viñas 1989; Fullola et al. 1990) que cabe sumar a las ya conocidas de este yacimiento (Fortea 1973), las piezas de Sant Gregori y Picamoixons -que carecen de contexto preciso- (García Díez et al. 1996) y la placa grabada de Forcas II (Utrilla y Mazo 1997).

Dentro de esta discusión, el hallazgo de un bloque de grandes dimensiones con grabados en Balma Guilanyà (Fig. 1) (Casanova et al. 2007 Martínez-Moreno et al. 2006a) puede ser relevante. La contextualización arqueo-estratigráfica y cronométrica de los bioindicadores y tendencias tecno-tipológicas del conjunto arqueológico asociado a esta manifestación artística define su adscripción crono-cultural. A tenor de estos resultados, se presentan posibles paralelos con otras manifestaciones parietales y mobiliares descritas en los últimos años en la zona mediterránea de la Península Ibérica, cuya atribución cronológica y/o cultural es imprecisa. Este resto incrementa el escaso catálogo de arte mueble conocido para este período, y proporciona claves con las que

(1) Román, D. 2010: El poblament del final del Plistocè en les comarques del nord del País Valencià a partir de l'estudi tecno-tipològic de la indústria lítica. Tesis doctoral inédita. Universitat de València.

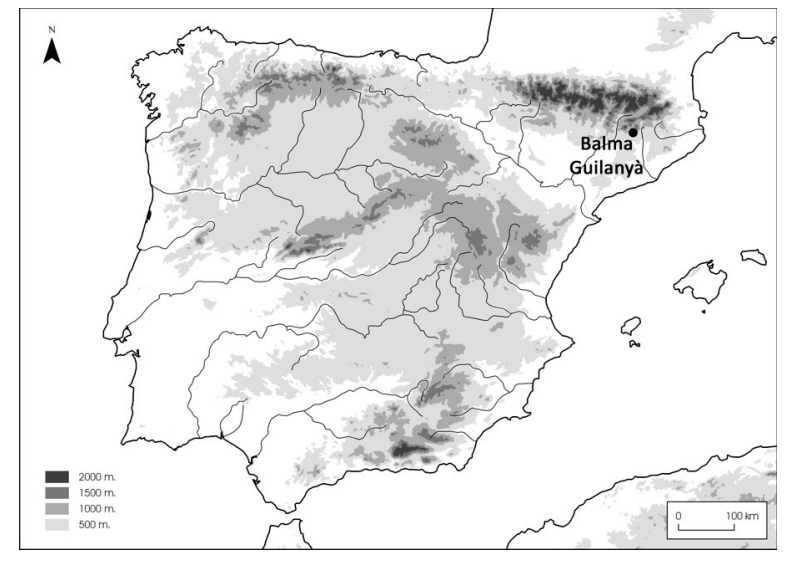

Fig. 1. Localización de Balma Guilanyà (Prepirineo de Lleida).

caracterizar las manifestaciones artísticas del Mesolítico.

\section{LA SECUENCIA HOLOCENA DE BALMA GUILANYÀ}

Balma Guilanyà (coordenadas $\mathrm{X}=385087$ $\mathrm{Y}=4660546$, UTM H31N ED50) es un abrigo que se localiza a 1157 m.s.n.m. en las primeras estribaciones del Prepirineo de Solsona (Serra de Busa, Lleida). Se conforma bajo una visera de conglomerados del sistema aluvial superior de Berga de edad oligocena (ICC 2008). Esta cornisa fue parcialmente afectada por la construcción de una pista forestal, que permitió a J. Castany y L. Guerrero su descubrimiento en 1992 (Fig. 2). Ese año se iniciaron trabajos dirigidos a evaluar su potencial arqueológico (Parcerisas et al. 2003). Entre 2001 y 2008 se han realizado excavaciones sistemáticas dentro del proyecto "El asentamiento humano en el Pirineo Oriental durante el Pleistoceno Superior y el Holoceno", y en la campaña del 2008 se recuperó el bloque grabado que presentamos en este artículo.

Este asentamiento se ubica en un pequeño valle construido por el arroyo de Ventolrà, un torrente de régimen nival que conecta el Pla de Busa -un altiplano en el techo de la Serra de Busa a $1.500 \mathrm{~m}$ de altitud- con las llanuras que bordean el Pirineo Oriental (Depresión central catalana y Valle del Ebro), abocando al Cardener, afluente del Llobregat.

En el depósito se diferencian dos unidades estratigráficas que corresponden a períodos cro- 


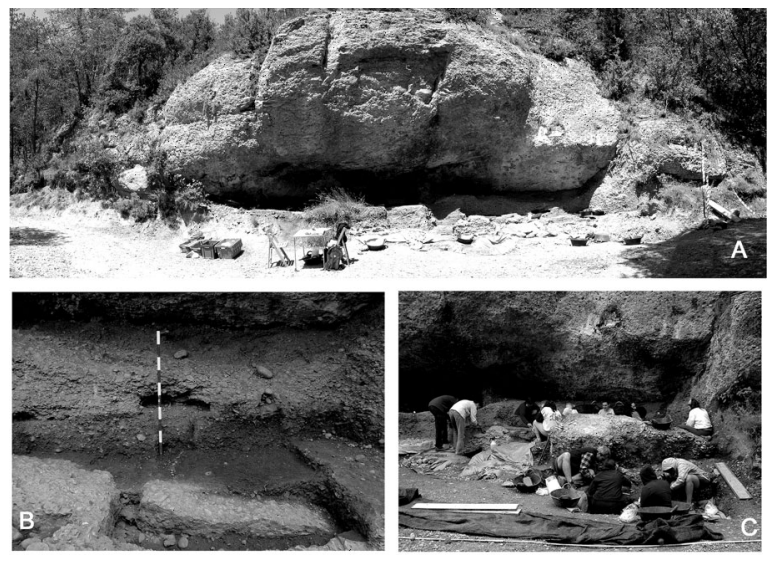

Fig. 2. Balma Guilanyà. A) Cornisa de conglomerados bajo la que se localiza el depósito. B) Secuencia sedimentaria holocena delimitada por caídas de la visera a techo y a base. C) Detalle de la excavación: en primer plano se aprecia la excavación de los niveles de la secuencia inferior (Tardiglaciar); en la parte posterior se observa la zona de caída de bloques que corresponde con la secuencia superior (Holoceno).

no-climáticos distintos: la unidad inferior radiométricamente se adscribe al ciclo Bolling/Allerod (2), mientras la unidad superior se conforma a lo largo del Holoceno inicial. Estos niveles sedimentarios están separados por una caída de la visera del abrigo que sella la secuencia inferior, y sobre la que asienta la serie holocena. A techo de la unidad superior se registra otra caída de bloques. Las características generales de las unidades arqueológicas de la secuencia inferior del abrigo han sido publicadas (Casanova et al. 2007; Martínez-Moreno et al. 2009), y en este artículo se contextualiza la posición arqueoestratigráfica, la cronometría, los indicadores bioarqueológicos, las características del tecno-complejo lítico y otros indicadores culturales de la unidad superior, en la que apareció la placa grabada.

\section{Arqueoestratigrafía de la unidad superior}

La sedimentación de la secuencia holocena se compone de una matriz arcillosa junto a elementos detríticos de pequeño tamaño y cantos, que

(2) $\mathrm{Al}$ referir los rangos cronométricos de las cronozonas climáticas Bolling/Allerod, Dryas Reciente, PreBoreal y Boreal nos remitimos al modelo cronoclimático GICC05, construido a partir de proxys de alta resolución de los núcleos de hielo NGRIP y GRIP de Groenlandia (Lowe et al. e INTIMATE group 2008). provienen de la alteración de los conglomerados de la cornisa. En la sección arqueoestratigráfica se posicionan las unidades arqueológicas definidas a partir de la dispersión vertical de los objetos coordenados en una sección transversal (Fig. 3). Varios desplomes de la cornisa inciden sobre la configuración del registro arqueológico. Pese a que aparentemente tiene un limitado desarrollo -no superior a $20 \mathrm{~cm}$ (Fig. 2)-, este espesor está condicionado por la caída de grandes bloques que provoca la compresión del paquete sedimentario.

La disposición vertical de los coordenados sugiere dos concentraciones en la densidad de artefactos, la primera $-\mathrm{C}$ - en la parte superior de la sección por debajo de la primera caída de la visera; la segunda $-\mathrm{C} 1$ - se posiciona por encima de la segunda caída de bloques (Casanova et al. 2007). Entre estas dos acumulaciones aparece material arqueológico diseminado resultante de bioturbaciones (especialmente la acción de raíces) que podría explicar ese fenómeno de dispersión vertical entre $\mathrm{C}$ y $\mathrm{C} 1$.

Estos indicadores contextuales advierten que la secuencia holocena configura un palimpsesto con una limitada resolución contextual que sería el resultado de un número indeterminado de ocupaciones que se suceden a lo largo de una escala temporal, que puede acotarse radiométricamente. Aunque en el registro arqueológico no son raros carbones, ni artefactos y restos óseos con alteraciones térmicas, no se han detectado estructuras de combustión.

El bloque grabado -que denominamos Guilanyà I- se localiza en la parte más occidental de la zona excavada y se inserta en la parte superior del depósito, dentro de la secuencia holocena (Fig. 3). Sus dimensiones y su posición estratigráfica -acotada por caídas de bloques a techo y a base- permiten su atribución a la unidad C. Este argumento será discutido posteriormente en detalle.

\section{Marco radiométrico}

Para esta parte del depósito se dispone de 7 dataciones $\mathrm{C}^{14}$ que se han calibrado a $2 \delta$, aplicando el modelo de Hulu que contiene el software CalPal (Weninger et al. 2007) y los intervalos cronométricos se expresan en años cal BP (Tab. 1). Las muestras datadas corresponden a 3 carbones, 2 fragmentos de cáscara de avellana y 2 sobre colágeno extraído de un molar de Capra 


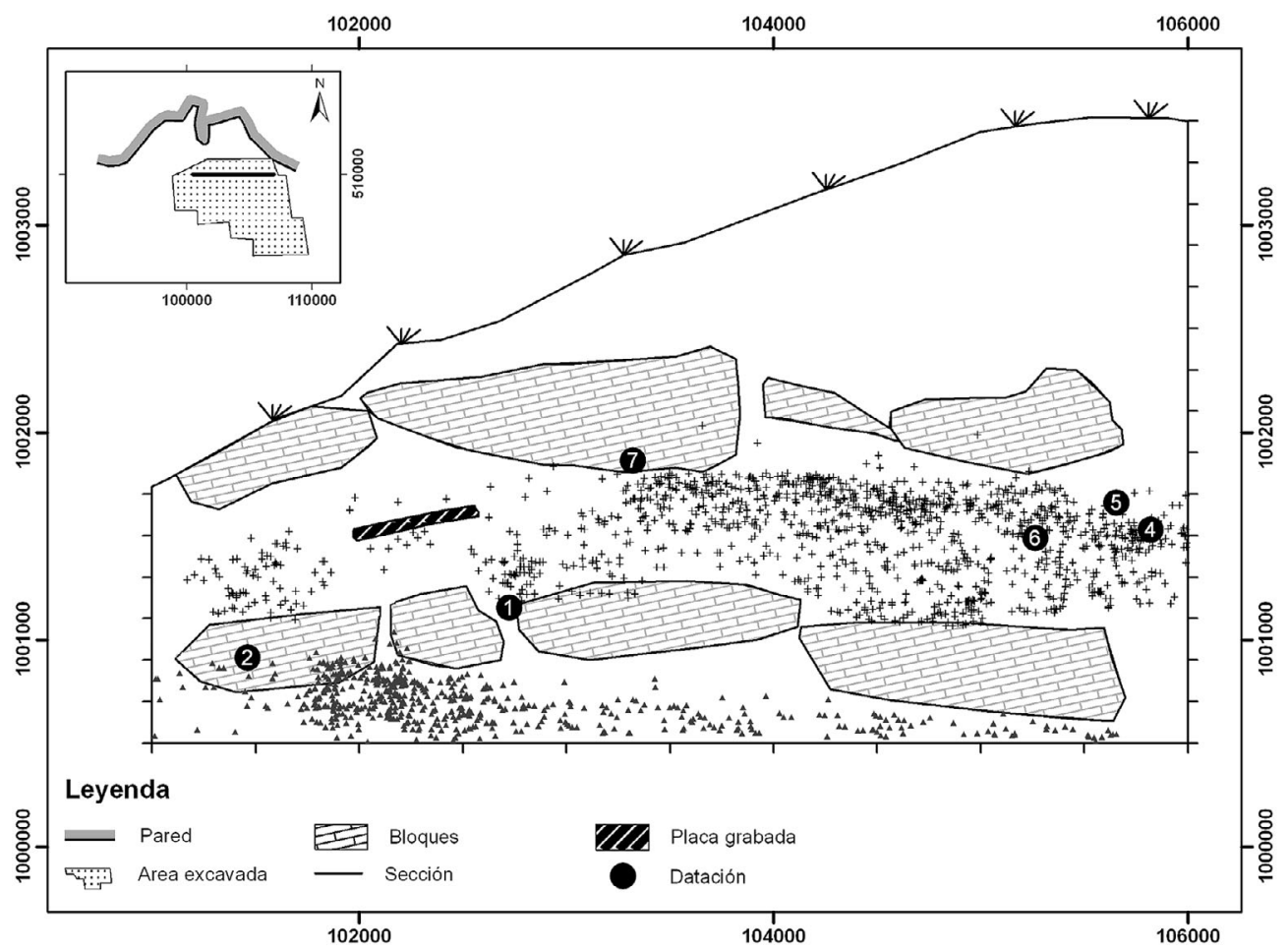

Fig. 3. Secuencia arqueoestratigráfica de Balma Guilanyà conformada a partir de la dispersión vertical (sentido E-O) de los materiales coordenados en el eje de $\mathrm{X}=101000-106000$ en el intervalo $\mathrm{Y}$ $=511000-511500$. Se posicionan las dataciones $C^{14}$ (cuya numeración remite a la tabla 1) para visualizar su relación con la placa grabada.

\begin{tabular}{|c|c|c|c|c|c|c|c|c|c|}
\hline Núm. & Nivel & Referencia & BP & $\sigma$ & Método & $\#$ & $\delta^{13} \mathbf{C}$ & cal BP (95\%) & Observaciones \\
\hline 1 & C1 & Beta-210728 & 9840 & 50 & AMS & Cor & $-25,5$ & $11360-11160$ & Cáscara Corylus \\
2 & C1 & Beta-186168 & 9410 & 60 & AMS & C & $-21,4$ & $10810-10490$ & Carbón aislado \\
3 & C & UBAR-368 & 8970 & 430 & CONV & C & $-24,8$ & $11250-9050$ & Carbón aislado \\
4 & C & Beta-185064 & 8680 & 50 & AMS & C & $-26,2$ & $9790-9510$ & Carbón aislado \\
5 & C & Beta-210730 & 8640 & 50 & AMS & Cor & $-24,3$ & $9740-9500$ & Cáscara Corylus \\
6 & C & Beta-252288 & 8180 & 50 & AMS & CB & $-20,0$ & $9330-8970$ & Diente Capra pyrenaica \\
7 & C & Beta-257402 & 7320 & 40 & AMS & CB & $-20,5$ & $8240-8000$ & Falange Equus caballus \\
\hline
\end{tabular}

Tab. 1. Serie $\mathrm{C}^{14}$ de la secuencia holocena de Balma Guilanyà y rangos calibrados cal BP a $2 \delta$ obtenidos por el modelo Hulu (Weninger et al. 2007). Las referencias de laboratorio en cursiva corresponden a fechas inéditas. Las muestras datadas (\#) son carbón (C), cáscara de Corylus avellana (Cor) y colágeno óseo (CB).

pyrenaica y una primera falange de Equus caballus. Aunque no retenemos la fecha UBAR-368 por la imprecisión de su intervalo cronométrico, los rangos calibrados de la serie se adscriben al Holoceno antiguo, y permite apreciar discontinuidades cronométricas (Fig. 4).

En los intervalos de las fechas sobre muestras vegetales y de colágeno óseo de la unidad C se observa una significativa asincronía cronomé- trica, del orden de 500-1500 años solares. Estas diferencias -a nuestro entender- advierten de la dificultad en la interpretación del registro radiométrico. Aunque podrían expandir significativamente el rango de ocupación humana del abrigo; alternativamente pueden relacionarse con el tipo de muestra datada. Se ha sugerido que el carbón puede "envejecer" el resultado de las fechas (el denominado efecto "madera vieja"). Contraria- 


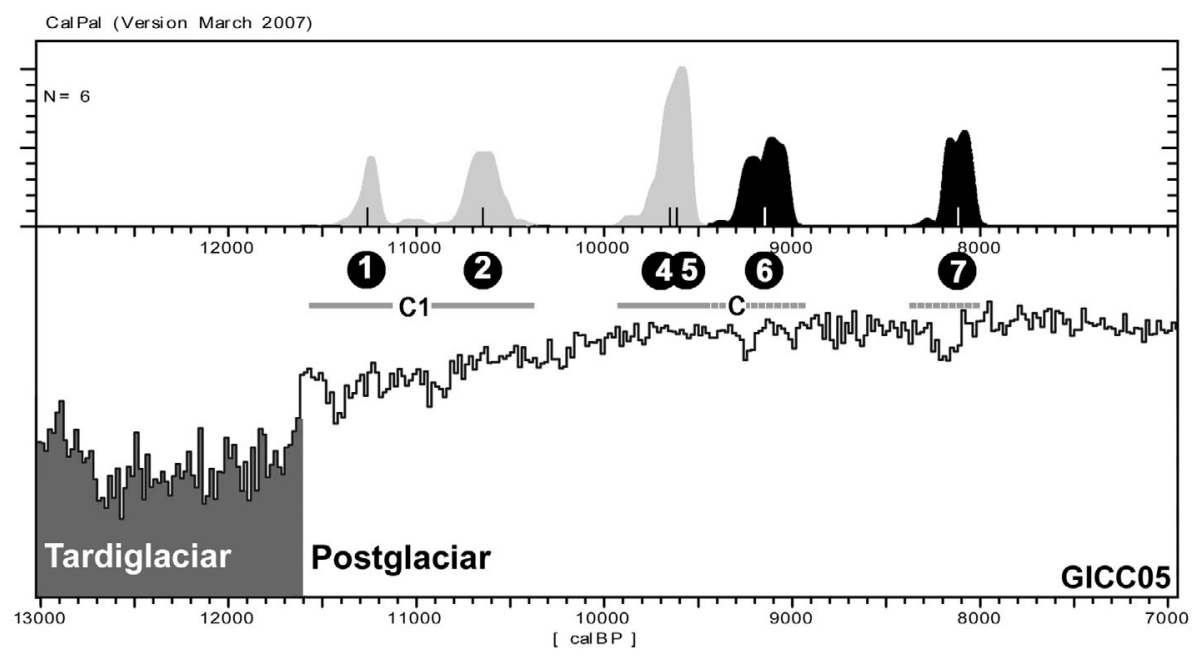

Fig. 4. Distribuciones gaussianas de los intervalos cronométricos calibrados (cal BP) de la serie holocena de Guilanyà y su correlación con el modelo cronoclimático GICC05 (Lowe et al. e INTIMATE group 2008). Se señala las asincronías en los rangos cronométricos obtenidos a partir de muestras vegetales y colágeno óseo (negro) del nivel C.

mente, las dataciones sobre hueso aunque son muestras de "vida corta", por problemas relacionados con la preservación del colágeno pueden proporcionar rangos temporales discordantes (Bronk-Ramsey 2008). Aunque actualmente no podemos evaluar la validez de estas inferencias, ya que carecemos de otros indicadores temporales independientes, señalamos que una muestra vegetal de vida corta tiene un rango cronométrico idéntico a otra de carbón (Beta-185064 y Beta-210730).

La serie radiométrica indica que el abrigo se reutiliza de forma intermitente durante el Holoceno antiguo, durante las cronozonas PreBoreal (C1) y Boreal (C). Estas ocupaciones se inician hacia el 11360 cal BP -poco después del límite Pleistoceno/Holoceno establecido en el $11600 \mathrm{cal}$ BP (Fiedrich et al. 2004)- y se extienden hasta el 8000 cal BP.

\section{Registro bioarqueológico e implicaciones ecológicas}

Los indicadores vegetales y animales de Balma Guilanyà informan del contexto ecológico de estas ocupaciones del Holoceno antiguo. El registro antracológico recuperado en las excavaciones recientes, completa y amplia el inventario taxonómico ya conocido (Parcerisas et al. 2003). En la secuencia holocena se han determinado más de
1.000 carbones, con una fuerte presencia de pino albar (Pinus tipo sylvestris) (más del $60 \%$ del total) al que se asocian taxones mesófilos como $\mathrm{Bu}$ xus sempervirens y rosáceas (Prunus y Maloidae). Por el contrario, en la secuencia tardiglaciar pino albar es hegemónico aunque se constata la presencia puntual de Juniperus, Betula y Prunus (E. Allué comunicación oral).

Este cambio denota la expansión del bosque caducifolio por entornos de montaña, en paralelo a la consolidación de las condiciones ambientales del Holoceno, un proceso que se detecta en los niveles postglaciares de Margineda en el Pirineo axial (Heinz y Vernet 1995) y por el nordeste de la Península Ibérica (Allué 2002). La presencia de rosáceas y la de Corylus y Quercus (estos últimos mal representados a partir de los carbones) sugiere la creciente importancia de la recolección de frutos silvestres, actividad de subsistencia característica del Mesolítico de la vertiente suroriental de los Pirineos (Martínez-Moreno et al. 2006b; Casanova et al. 2007).

La asociación de macromamíferos coincide con estas nuevas condiciones ambientales. Aunque la especie más abundante es Capra pyrenai$c a$ se aprecia un fuerte incremento de Cervus elaphus, fenómeno igualmente reconocido en los niveles de Holoceno antiguo de Margineda (Geddès 1995). Otras especies identificadas son Equus caballus, Sus scropha, Capreolus capreolus, Oryctolagus cunniculus, Vulpes vulpes, Felis 
sylvestris, Lynx sp. y Meles meles. Esta asociación sugiere un ecosistema forestal termófilo, apreciación que coincide con la información antracológica. Sorprende la presencia de caballo que no parece encajar con el entorno de montaña en el que se ubica el yacimiento. Se ha señalado que esta especie se rarifica al final Pleniglaciar, sin embargo, la fecha obtenida (Beta-257402) confirma su perduración en la vertiente surpirenaica durante el Holoceno antiguo.

\section{Contexto tecno-tipológico}

En este artículo sintetizamos algunas de las características tecno-tipológicas de los conjuntos líticos de la secuencia holocena (Martínez-Moreno et al. 2006a, 2006b, 2007; Casanova et al. 2007). Estos tecno-complejos se elaboran a partir de una amplia gama de rocas de escasa aptitud para la talla están presentes en los conglomerados de la visera del abrigo (cuarzos, cuarcitas o calizas) y que conforman más del $65 \%$ de los artefactos recuperados. El sílex, la segunda materia prima más empleada, puede tener un origen regional y su presencia se detecta en el Pirineo suroriental (Terradas 1995).

Diversos indicadores técnicos señalan que los instrumentos se elaboran en el asentamiento. Los sistemas de talla, esencialmente expeditivos, siguen una explotación unifacial a partir de plataformas naturales o con escasa preparación, obteniéndose lascas pequeñas y laminillas cortas que conforman módulos métricos de pequeño tamaño. En menor medida se identifican otros métodos como el discoide y la talla bipolar. No se reconocen sistemas laminares/lamelares y estos soportes no aparecen en los niveles de la secuencia superior (Casanova et al. 2007).

El escaso componente retocado microlítico (inferior a $3 \mathrm{~cm}$ ) se configura sobre lascas o fragmentos mayoritariamente de sílex. Excepto un triángulo fragmentado, no se han recuperado armaduras pequeñas sauveterrienses o armaduras largas tardenoides, indicadores empleados para su adscripción a las facies microlaminar o geométrica de los conjuntos post-paleolíticos peninsulares (Fortea 1973). En paralelo, se aprecia una fuerte reducción tipológica de los artefactos. La práctica totalidad de los retocados se adscriben al "fondo común" (denticulados, raspadores, muescas y piezas astilladas), artefactos habitualmente relacionados con las actividades domésticas en oposición a las armaduras, instrumental cinegético que no se detecta en el conjunto.

Se han recuperado más de 20 moluscos marinos: 7 Antalis sp., 1 Acantocardia sp., 1 Cyclope sp. y 13 Columbella rustica (Fig. 5). Recientemente hemos propuesto que Columbella es un identificador icónico de una red social durante el Holoceno antiguo que se articula en el valle del Ebro y la vertiente surpirenaica (Martínez-Moreno et al. 2010).

En resumen, los diferentes indicadores de orden cronológico y ecológico señalan que $\mathrm{C} 1$ y $\mathrm{C}$, pese la dilatada cronología de la serie radiométrica -entre 11300 y 8000 cal $\mathrm{BP}-$, se adscriben a una fase crono-climática del Holoceno antiguo que registra la incipiente configuración de un bosque termófilo, tras la consolidación de las nuevas condiciones ambientales que suceden a la crisis climática del Dryas Reciente.

Los atributos del conjunto lítico promueven su asignación a una tradición técnica expeditiva en la que predomina un instrumental aparentemente poco especializado y de carácter doméstico que se desarrolla durante el Postglaciar, y en el que están ausentes las armaduras. Estos atributos definen una nueva tradición cultural: el Mesolítico de muescas y denticulados. Esta tradición, que se formalizó en la reunión Epipaleolítico/Mesolítico en la Península Ibérica celebrada el 2005 en la Universidad del País Vasco-Vitoria (Alday (ed.) 2006), engloba un significativo número de asentamientos mesolíticos del valle del Ebro y la vertiente surpirenaica. No descartamos que esta respuesta técnica tenga una mayor distribución

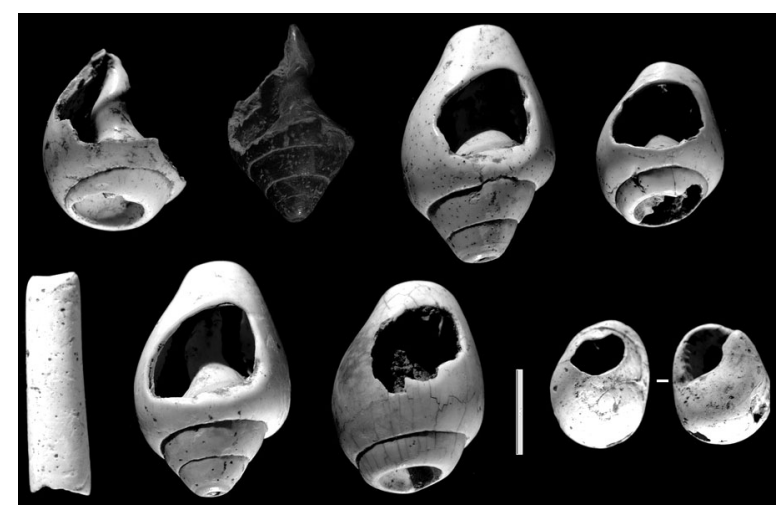

Fig. 5. Gasterópodos perforados (Columbella rustica y Cyclope sp.) y escafópodos (Antalis sp.) de Guilanyà C (Martínez-Moreno et al. 2010). Escala gráfica 0,5 cm. 
geográfica durante el Holoceno antiguo (Martínez-Moreno et al. 2007).

\section{LA PLACA GRABADA GUILANYÀ I}

En la parte superior de la secuencia holocena apareció un bloque de grandes dimensiones $(63,5 \times 50 \times 7,55 \mathrm{~cm})$ y $34 \mathrm{~kg}$ de peso, con una superficie muy irregular conformada por crestas y depresiones. Durante la excavación se identificó un motivo en forma de aspa que describimos más adelante (Fig. 6). Su tamaño y posición dentro del contexto sedimentario hacían improbable que fuera intrusivo, y asumimos que formaba parte de la unidad arqueológica. Aunque la materia prima del bloque (lutitas) se identifica en el entorno inmediato, no aparece en los conglomerados del abrigo. Esto implica que no es un grabado desprendido de la visera, sino que la placa debió ser recogida y transportada al abrigo durante el Holoceno antiguo.

Su posición altimétrica y su relación con las dataciones $\mathrm{C}^{14}$ permiten precisar su posible rango cronométrico (Tab. 1, Fig. 4). Las dataciones más antiguas (núms. 1 y 2) se asocian a la parte inferior de la secuencia, fechando la sub-unidad $\mathrm{C} 1$ en la cronozona PreBoreal. El bloque no está en contacto directo con los conglomerados que conforman la base de la secuencia holocena, se asienta sobre sedimentos del nivel C (Fig. 6), lo que
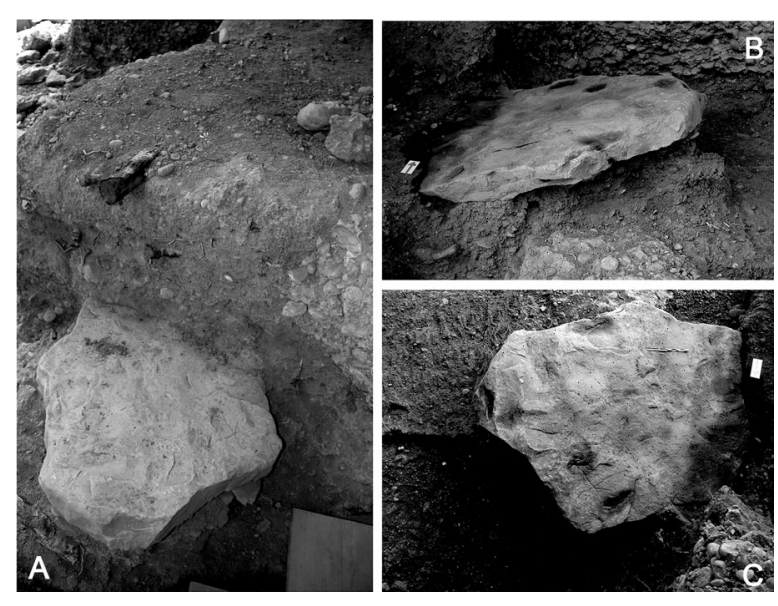

Fig. 6. La placa grabada Guilanyà I. A. Detalle de su excavación. B. Posición la placa interestratificada entre las caídas de bloques y su asiento sobre sedimento sobre el nivel C. C. Placa completamente excavada. En el ángulo inferior izquierdo se aprecia el motivo con forma de aspa. permite su relación con las fechas de la parte superior (núms. 4, 5, 6 y 7) dentro del intervalo 9800-8000 cal BP, en la cronozona Boreal.

A nuestro entender, los rangos que derivan las dataciones sobre colágeno óseo (especialmente la obtenida sobre la falange de caballo) se desvían excesivamente de la tendencia que marcan las fechas sobre restos vegetales. No descartamos que esta datación presente problemas relacionados con la conservación del colágeno, fenómeno que hemos observado al referir a la fecha Ua-34298 obtenida en los huesos humanos de este yacimiento (García Guixé et al. 2009; Martínez-Moreno y Mora 2009). En función de estas observaciones, proponemos ubicar la placa grabada dentro del rango comprendido entre 9800-9000 cal BP, durante el X milenio cal BP.

Los bordes del bloque no parecen trabajados y en general la pieza presenta una conservación muy desigual, aunque el área con grabados no está alterada. En la superficie grabada se observan trazos de origen mecánico -algunos recientes- que se reconocen por su pátina blanquecina, su mayor anchura y el tipo de sección (aplanada o redondeada). A falta de motivos figurativos, el bloque se ha orientado de manera arbitraria, haciendo coincidir la base con el eje de mayor anchura. Se pueden distinguir claramente trazos de surco fino y superficial que son dominantes, de otros más profundos y anchos obtenidos por el paso repetido del instrumento de grabado. Estos últimos se concentran en el cuadrante superior derecho de la pieza, formando un tema relativamente individualizado del resto (Fig. 7).

La erosión o alteración de la pieza se hace patente en la parte superior izquierda, dificultando el seguimiento e identificación del tema grabado. Las concreciones rellenan parcialmente algunos surcos. Un reticulado que abarca la mayor parte de la pieza se pierde en algunas zonas. Según la orientación dada, la reticulación dibuja un trazado rectangular con líneas verticales más separadas que las horizontales (Fig. 7). Sus límites no están bien definidos, no pudiendo considerarse un reticulado enmarcado.

Las zonas mejor conservadas del bloque se localizan en el ángulo inferior izquierdo y en la zona central y superior derecha. No podemos precisar si conforman un tema único o varios reticulados yuxtapuestos y articulados. La primera opción parece probable, ya que en su conjunto su ejecución es poco cuidada, sin regularidad en el 

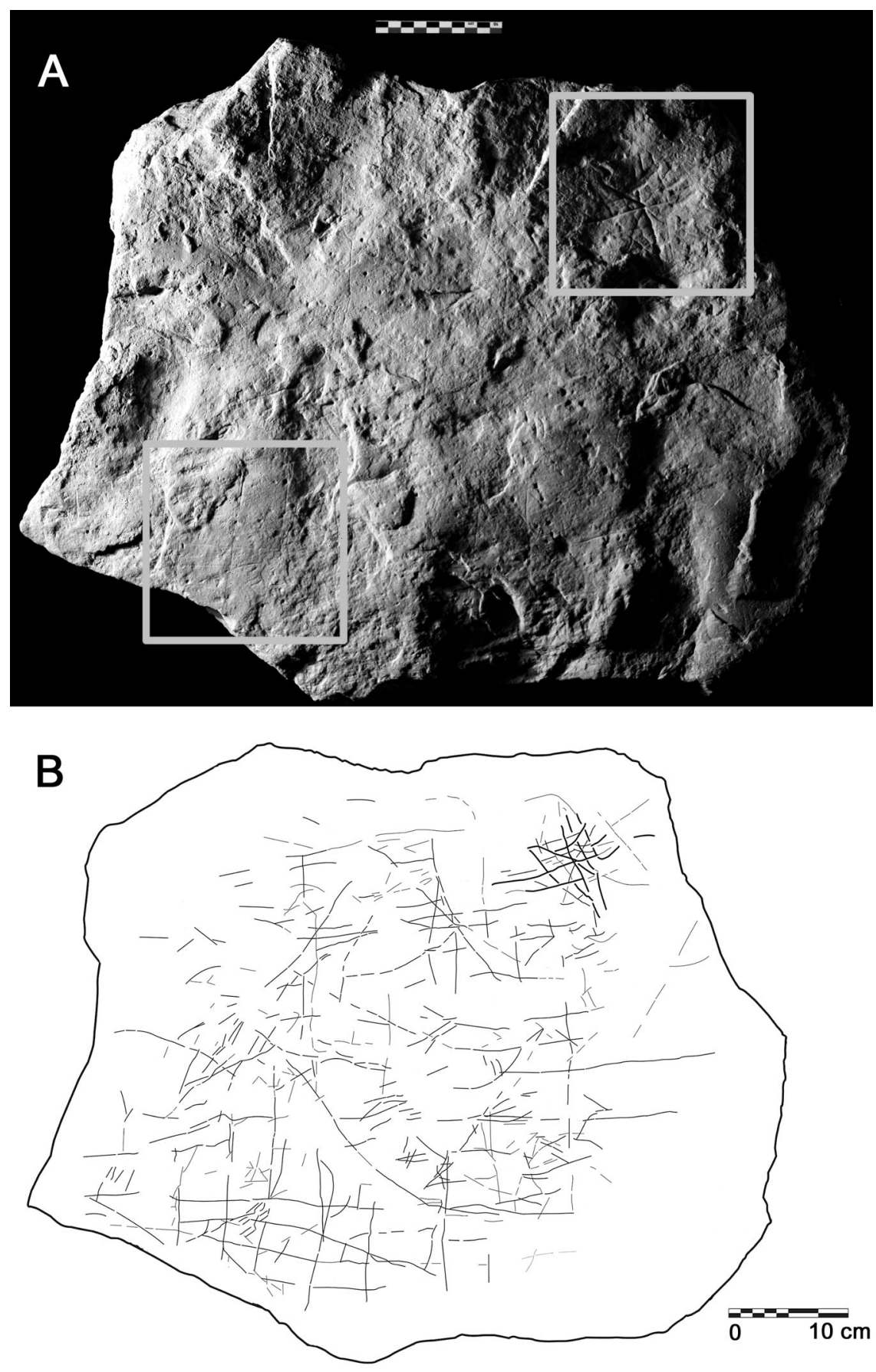

Fig. 7. Placa Guilanyà I. A. Vista general de la placa, en el ángulo superior derecho se aprecia el motivo en forma de aspa (Fig. 8A), y en el ángulo inferior izquierdo algunos de los trazos que conforman el reticulado (Fig. 8B). B. Relevamiento de la trama de líneas, retículas y motivos identificados en Guilanyà I (calco realizado por Mónica López i Prat).

trazado de las líneas y en la que no faltan rectificaciones en la orientación y en el recorrido de los trazos. Algunos surcos verticales y horizontales parecen abarcar la totalidad de la superficie. Que no se conserve ningún recorrido completo nos impide descartar que sea un tema compuesto mediante la adición de diversos reticulados (Fig. 8). Esta imprecisión genera la sensación de que el 

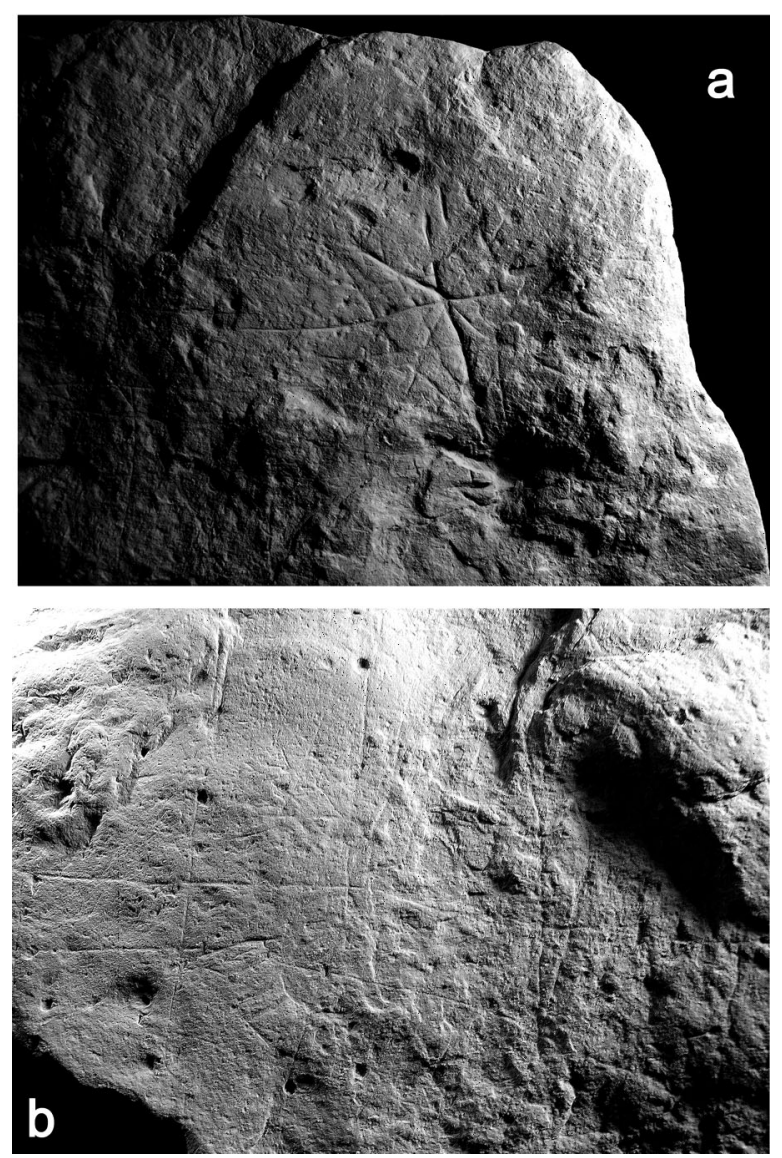

Fig. 8. A. Detalle del motivo es aspa situado en la parte superior derecha de la pieza. B. Detalle del reticulado en la parte inferior de la placa. La configuración de estos motivos sugiere una ejecución intencional con una finalidad simbólica o artística.

motivo de mayor extensión aunque se identifica con relativa facilidad, es de ejecución poco cuidada. Considerando el reticulado como un tema único, los trazos tienen cierta curvatura en su recorrido vertical y horizontal, y la distancia entre los surcos es variable no faltando rectificaciones especialmente en la parte inferior izquierda de la pieza.

En el ángulo superior derecho y superpuesto al reticulado, se individualiza un haz de líneas entrecruzadas en aspa de ocho brazos con un trazo de mayor recorrido (el horizontal izquierdo) (Fig. 8A). La anchura y profundidad de las líneas otorga unidad a este tema y algunas se desvían de su recorrido al cruzarse con otros trazos. Este motivo se superpone al reticulado, aunque varios trazos del aspa siguen la misma orientación que los del reticulado. Finalmente otros se alejan visiblemente del reticulado, en su mayoría surcos cortos y enmarañados o largos y curvilíneos, pero sin definir un motivo preciso, y aunque es posible que algunos sean de origen mecánico, presentan pátina.

A nuestro entender, esta composición no tiene un sentido funcional. Así, en un bloque de pizarra de grandes dimensiones $(36,4 \times 19,5 \mathrm{~cm})$ del nivel 4 de Filador se señaló una retícula o ajedrezado, que combinaba surcos longitudinales a los que se superponían trazos oblicuos y verticales muy regulares y estrechos, afectando a toda la superficie del soporte (Fullola-Pericot et al. 1990). Se ha propuesto que este patrón podría derivar del uso de esta placa como tabla para cortar (D’Errico y Possenti 1999). Sin embargo, esta posibilidad no explica los motivos representados en la placa Guilanyà I. Atributos como la irregular superficie del soporte, la delineación de los reticulados que combinan trazos ortogonales y otros curvos o el tema en forma de aspa, conforman motivos individualizados y deliberados (Figs. 7 y 8). Estas características contrastan por completo con la reiteración de trazos ortogonales y superpuestos que sustentan el carácter funcional inferido para la mencionada placa de Filador.

\section{MANIFESTACIONES ARTÍSTICAS POST-PALEOLÍTICAS DE LA PENÍNSULA IBÉRICA}

El bloque de Balma Guilanyà presenta un tema grabado de carácter no figurativo relativamente sencillo que asocia un reticulado, un motivo en aspa y trazos sueltos rectos y curvos. Esta composición es interesante al encuadrarse en un período en el que, hasta la fecha, sólo se había documentado la grafía mobiliar del nivel II de Filador (excavaciones de Vilaseca 1949). Dada su notoria coincidencia temática, su valoración conjunta parece pertinente.

La pieza decorada de Filador es de pizarra y de menores dimensiones $(27,1 \times 24,8 \mathrm{~cm})$ que la de Guilanyà. Forma parte de un conjunto de piezas procedente de los niveles IV y VII sin motivos decorativos definidos (Fullola et al. 1986; García Argüelles et al. 2005). El ejemplar al que nos referimos posee un tema geométrico relativamente complejo en el que se distinguen varios reticulados y alguna serie de trazos paralelos junto 
a haces de líneas de trazado irregular o entrecruzado. Este resto se encuadra en un nivel caracterizado por la abundancia de denticulados (Fortea 1973; García Argüelles y Fullola 2006). En ambas piezas, la decoración cubre la práctica totalidad de la superficie y el tema principal está formado por reticulados, lo que permite reconocer ciertas semejanzas entre ellas, a pesar de la diferencia en los soportes y tamaños. Admitiendo que son escasas las manifestaciones artísticas para la etapa en la que se encuadran estas piezas, su similitud temática y cronológica permite considerar estos motivos como propios del Mesolítico de muescas y denticulados.

Si se amplía la discusión a las piezas grabadas de etapas precedentes (Epipaleolítico microlaminar), observamos que continúa la tradición gráfica magdaleniense y las industrias no sauveterrienses mantienen los mismos procedimientos técnicos y temáticos del Magdaleniense superior y final. Esta cuestión ha sido señalada en las piezas de arte mueble de Molí del Salt (García Díez y Vaquero 2006) y de otros yacimientos magdalenienses del Mediterráneo, en especial Parpalló (Villaverde 2004).

En su estudio sobre un par de piezas de Sant Gregori de la colección Rodón del Museu de Alcover (Tarragona) García Díez et al. (2003) evalúan el panorama del arte epipaleolítico/mesolítico mediterráneo, especialmente de Cataluña. Señalan que, en las piezas que se correlacionan con la tradición microlaminar, se aprecian procedimientos de ejecución y temáticas relacionadas con el arte del Magdaleniense superior-final del Mediterráneo ibérico. Por el momento, estas representaciones figurativas muebles no se asocian a tecno-complejos sauveterroides. Pero faltan criterios con los que establecer la cronología de algunas manifestaciones parietales de la zona septentrional valenciana $\mathrm{y}$, de hecho, la precisión cronológica o contextual de buena parte de la documentación arqueológica disponible es limitada.

Una novedad sugerente es la asignación a este período de algunas representaciones parietales descritas en las cuencas altas de la Rambla Carbonera y del Riu de les Coves, en Castellón (Guillem et al. 2001; Martínez Valle et al. 2003, 2009). A raíz del descubrimiento del Abric d'en Melià se ha incrementado el inventario de abrigos con grafías de diversa índole y que comparten la presencia de representaciones zoomorfas con diversos grados de estilización/simplificación. No puede descartarse que esa variación de motivos denote cierta amplitud cronológica. En algunos conjuntos conviven distintas ejecuciones en las representaciones zoomorfas de diferentes paneles o dentro del mismo panel pero, en este caso, las más simplificadas y geométricas ocupan zonas periféricas. Sin querer aventurar la amplitud cronológica a la que pueden remitir estas manifestaciones - difícil de evaluar sin su correlación con arte mueble-, indicamos que algunas variantes tienen paralelos con piezas del arte mueble regional y con temas parietales de otras zonas peninsulares que se han adscrito al denominado "estilo V" (Bueno et al. 2007; Alcolea y Balbín 2007). La ausencia de representaciones figurativas, sea cual sea su grado de simplificación o geometrismo, en contextos en los que se identifican tecno-complejos sauveterroides y del Epipaleolítico geométrico, sugiere que su límite se ubica en la tradición microlaminar de filiación magdaleniense.

Dentro del arte mobiliar del ámbito mediterráneo ibérico, la variedad de soluciones a la hora de ejecutar figuras zoomorfas, oscila desde representaciones muy parecidas al arte mueble de Molí del Salt (García Díez 2004; García Díez y Vaquero 2006) o Sant Gregori (Vilaseca 1933; Fullola et al. 1990) a otras sin un correlato directo con las hasta ahora conocidas. Las primeras se caracterizan por cuerpos alargados con cuellos estrechos y exageradamente proyectados hacia delante, y cabezas pequeñas de tendencia triangular pero de clara voluntad naturalista. Pueden citarse algunas del Abric d'en Melià o la figura pintada del Abric de Bovalar, una imagen prácticamente especular de la conocida cierva grabada de la plaqueta de Sant Gregori. Las segundas tienen cuerpos de tendencia naviforme, muy simplificados y desproporcionados, prestando escasa atención a los detalles de cabeza y patas, muchas veces reducidas a simples trazos lineales. Hay algunos ejemplos en Melià (Guillem et al. 2001; Martínez Valle et al. 2003), Cova del Bovalar (Martínez Valle et al. 2009) o en el Abric del Cingle (Guillem y Martínez Valle 2009).

Las figuras de carácter naturalista se correlacionan con piezas de arte mueble que contextualmente se adscriben a industrias de tradición magdaleniense (bien sean del Tardiglaciar o del inicio del Holoceno). Consideramos significativo que las figuras del componente más simplificado y geométrico no se encuadren en ese contexto in- 
dustrial. Sin embargo, en los conjuntos parietales citados se inventarían motivos geométricos que podrían encontrar paralelos en contextos epimagdalenienses y mesolíticos, como un reticulado oblicuo de una de las piezas sin contexto de Sant Gregori o los reticulados de Guilanyà y Filador, en principio cronológicamente más tardíos.

Un tema particular es el zigzag bien definido asociado a un ciervo de tendencia naturalista, aunque descuidado en sus extremidades, del Abric del Cingle del Barranc de l'Espigolar que remite de nuevo a la tradición magdaleniense. Esta grafía nos proporciona un argumento más para vincular la tradición gráfica parietal de la zona castellonense con el arte de las industrias microlaminares de cronología holocena atribuidas al Epimagdaleniense. En cualquier caso, los signos a los que estamos haciendo referencia y las líneas entrecruzadas dominan el apartado gráfico de la mayor parte de los conjuntos parietales del norte de Castellón. Por su amplia cronología, su valoración ha de hacerse en los mismos términos en los que Barandiarán (1987) analizó la temática del arte mesolítico geométrico de Cocina.

Contamos con escasas piezas y referidas a pocos yacimientos del horizonte industrial posterior a la cronología propuesta para la placa Guilanyà I, que se correspondan con el Epipaleolítico geométrico: las plaquetas grabadas de la Cueva de la Cocina (Pericot 1945; Fortea 1971, 1973), una plaqueta del Abrigo de las Forcas II (Utrilla y Mazo 1997) o una pieza de la Cueva de la Torca (Villaverde et al. 2000) cuya adscripción, como veremos, es imprecisa.

La colección de la Cueva de la Cocina está formada por más de 30 plaquetas, la mayor parte grabadas, en ocasiones grabadas y pintadas o simplemente pintadas. Se encuadran en los niveles precerámicos atribuidos al Epipaleolítico geométrico o fase Cocina II (Pericot 1945; Fortea 1971, 1973). Aunque el conjunto carece de un estudio global su caracterización es posible a partir de las piezas publicadas (Pascual 2008). Son de dimensiones reducidas -la mayoría entre 7-10 $\mathrm{cm}-$, algunas de contorno irregular, otras ovales o redondeadas. Normalmente se decoran por una cara, si bien algunas lo están por ambas superficies. La temática del grabado es lineal con una tendencia a abarcar la totalidad de la superficie, aunque en algunas la zona grabada es menor. Dominan los haces de líneas paralelas con orientaciones distintas, en ocasiones rellenas de trazos cortos que dan lugar a motivos escaleriformes. En otros casos, las bandas paralelas se articulan con líneas oblicuas paralelas. Es llamativa la variedad de temas decorativos y la escasa repetición de formas, que denota que, aunque no exista una estandarización temática se intenta cubrir la superficie con haces y líneas paralelas. En los diarios de excavación de Pericot y en los publicados por Fortea, al menos un par presentan bandas quebradas de trazado múltiple, alguna articulación de bandas escaleriformes y algún reticulado.

En la parte inferior del nivel II de Forcas II se recuperó un fragmento de plaqueta de arenisca de pequeñas dimensiones $(4,4 \times 3,3 \mathrm{~cm})$ con un grabado somero, que se asocia con triángulos y con la técnica del microburil que remiten al Epipaleolítico geométrico tardío datado en $7240 \pm 40 \mathrm{BP}$ (Utrilla y Mazo 1997). La decoración cubre toda la superficie y configura un motivo geométrico compuesto por tres líneas paralelas verticales que forman bandas divididas por series de líneas horizontales paralelas.

La plaqueta grabada de la Cueva de la Torca (Villaverde et al. 2000) fue localizada en un lote de materiales recogidos en superficie que incluía algún microlito geométrico, dorsos de tradición microlaminar y materiales cerámicos por lo que, aunque este motivo decorativo es interesante, su cronología es incierta. Esta pequeña placa de esquisto $(5 \times 3 \mathrm{~cm})$ está decorada por las dos caras. En una se aprecia una serie de seis bandas de líneas paralelas (dos rellenas de trazos oblicuos que definen un signo escaleriforme), un motivo en espiga y un haz de líneas paralelas formando una banda. La temática de la otra cara es parecida y combina bandas paralelas y escaleriformes, en este caso flanqueadas por una banda de líneas paralelas quebradas parcialmente rellenas de trazos cortos. Aunque el motivo es coherente con una adscripción Epimagdaleniense/Epipaleolítico microlaminar con paralelos en el ciclo artístico magdaleniense, no puede negarse cierta similitud con la plaqueta de Forcas II, en la que las bandas paralelas ordenadas en series abarcan la totalidad de la superficie.

Una vez analizados el limitado registro mueble conocido y el arte parietal hasta ahora publicado en la zona de Castellón, podemos concluir que la presencia exclusiva de temas geométricos con ausencia de elementos figurativos se asocia únicamente al arte mueble adscrito al Epipaleolítico sauveterroide, al Mesolítico de muescas 
y denticulados y al Epipaleolítico geométrico. Esta circunstancia contrasta claramente con el arte mueble de tradición magdaleniense y con el arte parietal epipaleolítico de tradición paleolítica descrito en Castellón, en los que abundan temas figurativos y zoomorfos con diversos estados de estilización y geometrismo.

\section{PERSPECTIVAS DE FUTURO}

En resumen, aunque escasas, cuando se analizan globalmente las manifestaciones artísticas finipaleolíticas y mesolíticas de la vertiente mediterránea ibérica, no pueden establecerse soluciones de continuidad. Bajo esta perspectiva, el grabado Guilanyà I confirma la entidad del fenómeno gráfico en la zona septentrional del ámbito mediterráneo del horizonte del Mesolítico de muescas y denticulados, y abre interesantes perspectivas al corresponder a un período en el que las manifestaciones artísticas no son abundantes.

Al inicio de este artículo nos hacíamos eco de los problemas de orden cronológico, contextual y estilístico que han dificultado la caracterización del denominado "arte del final del ciclo artístico paleolítico" (D’Errico y Possenti 1999), que puede prolongarse durante el Holoceno. En los últimos años, esta situación se ha modificado con nuevos hallazgos de arte mueble crono-culturalmente contextualizado. Para algunos autores los paralelos establecidos entre estas manifestaciones artísticas identifican el denominado "estilo V" (Bueno et al. 2007). Las convenciones de este sistema gráfico encuentran su ascendente y tienen continuidad con el arte del final del Paleolítico superior, reconociéndose en el Mediterráneo occidental por lo menos desde el Magdaleniense (Villaverde et al. 2009). El "estilo V" se asigna al ámbito Epimagdaleniense/Epipaleolítico microlaminar, aunque a partir del registro cronométrico conocido estos restos se adscriben al ciclo Bolling/Allerod y Dryas Reciente, es decir al Tardiglaciar.

La placa grabada Guilanyà I no continúa con este sistema de grafías y los motivos representados tienden hacia grafías simples y sencillas - si se prefiere abstractas (aspas, reticulados, trazos sin aparente organización)-, son conocidas en contextos crono-culturales previos. Sin embargo, el interés que encierra este hallazgo es que remite a una fase posterior al señalado por el "estilo V".
Los indicadores arqueo-estratigráficos, cronométricos y bioarqueológicos permiten adscribir la placa Guilanyà I al Holoceno antiguo. La contextualización de este hallazgo dentro de un rango radiométrico preciso -el grabado no puede ser datado directamente-, aun a riesgo de equivocarnos, apunta como bastante probable el X milenio cal BP, en la cronozona Boreal. Igualmente, los atributos tecno-tipológicos de los conjuntos líticos de esta secuencia son característicos del Mesolítico de muescas y denticulados (Casanova et al. 2007), tradición que parece desarrollarse en el valle del Ebro y la vertiente surpirenaica durante el PreBoreal y Boreal (Alday (ed.) 2006).

Es decir, son motivos propios del arte mesolítico y su contextualización crono-cultural deriva argumentos para investigar algunos interrogantes reseñados en la introducción de este artículo. Bajo esta perspectiva consideramos que la placa grabada de Guilanyà abre nuevas perspectivas con las que analizar un rasgo tan propio de nuestra especie como es la necesidad de plasmar conceptos e ideas sobre soportes materiales, comportamiento que está en la definición de lo que entendemos por arte.

\section{AGRADECIMIENTOS}

Originariamente Marcos García Díez se hizo cargo del estudio de este resto, advirtiéndonos del interés de su hallazgo. Ethel Allué (IPHES, Tarragona), responsable del análisis antracológico, nos ha avanzado datos relevantes para la caracterización ecológica de la secuencia Holocena. Susana Vega, Jezabel Pizarro, Maria Lou y Joel Casanova (CEPAP-Universitat Autònoma de Barcelona) nos proporcionaron observaciones esenciales para la contextualización precisa de esta manifestación artística. Destacamos por su utilidad en este estudio, la calidad del calco realizado por Mónica López i Prat (CEPAP-Universitat Autònoma de Barcelona).

Reiteramos nuestro agradecimiento a la familia Guilanyà por permitirnos investigar en Balma Guilanyà desde 1992. Esta excavación está financiada por el Servei d'Arqueología i Paleontología-Generalitat de Catalunya y sus resultados se integran dentro del proyecto "El asentamiento humano en el Pirineo Oriental durante el Pleistoceno Superior y el Holoceno" (HUM2007-60317/ HIST y HAR2010-15002) del Ministerio de 
Ciencia e Innovación. Ésta es una publicación del grupo de investigación Cultura mediambiental $i$ comportament humà de la Universitat Autònoma de Barcelona, reconocido por la AGAUR (SGR729). Rafael Mora agradece el apoyo del Programa ICREA-Acadèmia.

\section{BIBLIOGRAFÍA}

Alcolea, J. y Balbín, R. 2007: "C14 et style. La chronologie de l'art pariétal à l'heure actuelle". L'Anthropòlogie 111: 435-466.

Alday A. (ed.) 2006: El Mesolítico de muescas y denticulados en la cuenca del Ebro y el litoral mediterráneo peninsular. Memorias de Yacimientos Alaveses 11. Diputación Foral de Álava. Vitoria.

Allué, E. 2002: Dinámica de la vegetación y explotación del combustible leñoso durante el Pleistoceno Superior y el Holoceno del Noreste de la Península Ibérica a partir del análisis antracológico. Tesis Doctoral. Universitat Rovira i Virgili, Tarragona. http://www.tdx.cesca.es/ (consulta 15-III-2010).

Barandiarán, I. 1987: "Algunos temas no figurativos del arte mueble prehistórico (A propósito de las placas grabadas de La Cocina)". Homenaje a D. Domingo Fletcher. Archivo de Prehistoria Levantina XVII: 59-80.

Bueno, P.; Balbín, R. y Alcolea, J. 2007: "Style V dans le bassin du Douro. Tradition et changement dans les graphies des chasseurs du Paléolithique Supérieur européen". L'Anthropologie 111: 549-589.

Bronk Ramsey, C. 2008: "Radiocarbon dating: revolutions in understanding". Archaeometry 50: 249275.

Casanova, J.; Martínez-Moreno, J. y Mora, R. 2007: "Traçant l'ocupació dels Pirineus: Balma Guilanyà i els caçadors-recol.lectors del Tardiglacial i l'Holocé antic al Prepririneu Oriental". Tribuna d'Arqueologia 2006: 59-83.

Cava, A. 2004: "Los procesos culturales del comienzo del Holoceno en la Cuenca del Ebro y su contextualización". Saldvie 4: 17-40.

D’Errico, F. y Possenti, L. 1999: “L’art mobilier épipaléolithique de la Mediterranée occidentale: comparaisons thématiques et technologiques". En D. Sacchi (ed.): Les facies leptolithiques du nord-ouest méditerranéen: milieux naturels et culturels. XXIVe Congrès Prehistorique de France, Carcassone 1994: 93-116. Carcassone.

Fortea, J. 1971: La cueva de La Cocina. Ensayo de cronología del Epipaleolítico (Facies Geométricas). Serie de Trabajos Varios del Servicio Investigaciones Prehistóricas 40. Valencia.
Fortea, J. 1973: Los complejos microlaminares y geométricos del Epipaleolítico mediterráneo español. Universidad de Salamanca. Salamanca.

Friedrich, M.; Remmele, S.; Kromer, B.; Hofmann, J.; Spurk, M.; Felix Kaiser, K.; Orcel, C. y Küppers, M. 2004: "The 12,460-Year Hohenheim oak and pine tree-ring chronology from Central Europe: a unique annual record for radiocarbon calibration and paleoenvironment reconstructions". Radiocarbon 46: 1111-1122.

Fullola-Pericot, J. M. y Couraud, C. 1984: "Le galet peint de l'abri du Filador". L'Anthropologie 88: 119-123.

Fullola-Pericot, J. M. y Viñas, R. 1989: "Dernières découvertes dans l'art préhistorique de Catalogne (Espagne)". L'Anthropologie 92: 123-132.

Fullola-Pericot, J. M.; Viñas, R. y García Argüelles, P. 1990: "La plaque en ardoise gravée de l'abri de El Filador (Catalogne, Espagne)". Cahiers Ligures de Préhistoire et de Prototohistoire, nouvelle série 3: 147-156.

García Argüelles, P.; Nadal, J. y Fullola-Pericot, J. M. 2005: "El Abrigo del Filador (Margalef de Montsant, Tarragona) y su contextualización cultural y cronológica en el Nordeste Peninsular". Trabajos de Prehistoria 62 (1): 65-83.

García Díez, M.; Fontanals, M. y Zaragoza, J. 2003: "Dues noves peçes gravades del jaciment de Sant Gregori (Falset, Tarragona): La col.lecció Ramón Rodón del Museu Municipal d'Alcover (Alt Camp)". Pyrenae 33-34: 165-174.

García Díez, M.; Rossel, J.; Vallverdú, J. y Vergès, J. M. 1996: "L'abric de Picamoixons i les estratègies dels darrers caçadors recol.lectors epipaleolítics". Quaderns de Vilaniu 29: 91-100.

García Díez, M. y Vaquero, M. 2006: "La variabilité graphique du Molí del Salt (Vimbodí, Catalogne, Espagne) et l'art mobilier de la fin du Paléolithique supérieur à l'est de la Péninsule Ibérique". L'Anthropologie 110: 453-481.

García Guixé, E.; Martínez-Moreno, J.; Mora, R.; Núñez, M. y Richards, M. P. 2009: "Human and animal stable isotope analysis from late Upper Palaeolithic site of Balma Guilanyà, Southeastern Pre-Pyrenees, Spain". Journal of Archaeological Science 36: 1018-1026.

Geddès, D. 1995: "La fauna mesolítica i neolítica de la Balma de la Margineda". En J. Guilaine y M. Martzluff (ed.): Les excavacions a la Balma de la Margineda (1979-1991) III. Ministeri d'Afers Socials i Cultura. Andorra: 83-97.

Guillem, P. M.; Martínez Valle, R. y Melià, F. 2001: "Hallazgo de grabados rupestres de estilo paleolítico en el norte de la provincia de Castellón: el Abric d'en Melià (Serra d'en Galceran)". Saguntum-PLAV 33: 133-140. 
Guy, E. 1993: “Enquête stylistique sur l'expression figurative Épipaléolithique en France: de la forme au concept". Paleo 5: 333-373.

Heinz, C. y Vernet, J. L. 1995: Anàlisi antracològica dels nivells mesolítics i del neolític antic de la Balma Margineda. Paleoecologia i relacions home-vegetació. En J. Guilaine y M. Martzluff (ed.): Les excavacions a la Balma de la Margineda (19791991). III. Ministeri d'Afers Socials i Cultura. Andorra: 26-64.

ICC 2008: Full 35-11. Institut Cartogràfic de Catalunya. http://www.icc.es/ (consulta 15-III-2010).

Lowe, J. J.; Rasmussen, S.; Björck, S.; Hoek, W.; Steffensen, J.; Walker, M. y Yu, Z. \& the INTIMATE group 2008: "Synchronisation of palaeoenvironmental events in the North Atlantic region during the Last Termination: a revised protocol recommended by the INTIMATE group". Quaternary Science Reviews 27: 6-17.

Martínez-Moreno, J.; Martzluff, M.; Mora, R. y Guilaine, J. 2006a: "D'une pierre deux coups: entre percussion posée et plurifonctionnalté. Les poids des comportements 'opportunistes' dans l'Epipaléolithique-Mésolithique pyrénéen”. En L. Astruc, F. Bon, V. Léa, P. Y. Milcent, S. Philibert (ed.): Normes techniques et pratiques sociales. De la simplicité des outillages pré- et protohistoriques. Éditions APDCA. Antibes: 147-160.

Martínez-Moreno, J. y Mora, R. 2009: "Balma Guilanyà (Prepirineo de Lleida) y el Aziliense en el Nordeste de la Península Ibérica". Trabajos de Prehistoria 66 (2): 45-60.

Martínez-Moreno, J.; Mora, R. y Casanova, J. 2006b: "El Mesolítico de los Pirineos surorientales: una reflexión sobre el significado de las 'facies de fortuna' del Postglaciar". En A. Alday (ed.): El Mesolitico de muescas y denticulados en la cuenca del Ebro y el litoral mediterráneo peninsular. Memorias de Yacimientos Alaveses 11. Gasteiz: 163-192.

Martínez-Moreno, J.; Mora, R. y Casanova, J. 2007: "El contexto cronométrico y tecno-tipológico durante el Tardiglaciar y Postglaciar de la vertiente sur de los Pirineos orientales". Revista d'Arqueologia de Ponent 16-17: 7-44.

Martínez-Moreno, J.; Mora, R. y Casanova, J. 2010: "Lost in the mountains?: Marine ornaments in the Mesolithic of the northeast of the Iberian Peninsula”. En E. Álvarez-Fernández y D. Carvajal (ed.): Not only Food. Marine, Terrestrial and freshwater molluscs in archaeological sites. Munibe suplemento 31. Sociedad de Ciencias Aranzadi. Donostia: $100-109$.

Martínez Valle, R.; Guillem, P. M. y Villaverde, V. 2003: "Las figuras grabadas de estilo paleolítico del Abric d'en Melià (Castelló): Reflexiones en torno a la caracterización del final del arte paleolítico de la España Mediterránea”. En R. de Balbín y
P. Bueno (eds.): El Arte Prehistórico desde los inicios del siglo XXI (I Symposium Internacional de Arte Prehistórico de Ribadesella 2002): 279-290. Ribadesella.

Martínez Valle, R.; Guillem, P. M. y Villaverde, V. 2009: "Grabados rupestres de estilo paleolítico en el Norte de Castellón”. R. de Balbín (ed.): Arte Prehistórico al aire libre en el Sur de Europa. Junta de Castilla y León. Salamanca: 225-236.

Olaria, C. 2008: Grafismo mobiliar magdaleniense de Cova Matutano (Vilafamés, Castellón) en el contexto del Mediterráneo peninsular. Monografies de Prehistòria i Arqueologia Castellonenques 7. Castellón.

Parcerisas, J.; Mora, R.; Pallarés, M. y Martínez, J. 2003: "Balma Guilanyà (Navès, Solsonès)". En Jornades d'Arqueologia i Paleontologia-2000. Comarques de Lleida (Lleida): 73-90. Barcelona.

Pascual, J. L. 2008: "La Cueva de la Cocina y el arte Epipaleolítico". Arqueología en Blanco y Negro. La Labor del Servicio de Investigaciones Prehistóricas 1927-1950: 183-188.

Pericot, L. 1945: "La cueva de la Cocina. Dos Aguas. Nota preliminar". Archivo de Prehistoria Levantina II: 39-75.

Ripoll, S. y Cacho, C. 1987: “Art mobilier du Paléolithique méditerranéen espagnol. Quelques nouvelles techniques". En J. Clottes (ed.): L'art des objets au Paléolithique 1-L'art mobillier et son contexte (Actes du Colloque de Foix-Le Mas d'Azil, 1987): 287-293. París.

Terradas, X. 1995. Las estrategias de gestión de los recursos líticos del Prepirineo catalán en el IX milenio BP. Treballs d'Arqueologia 3. Universitat Autònoma de Barcelona. Bellaterra.

Utrilla, P. y Mazo, C. 1997: "La transición del Tardiglaciar al Holoceno en el alto Aragón: los abrigos de Las Forcas (Graus, Huesca)". II Congreso de Arqueología Peninsular (Zamora, 1996) I Paleolítico y Epipaleolítico: 349-366. Zamora.

Vilaseca, S. 1934: “L'estació-taller de sílex de Sant Gregori". Memoria de la Academia de Ciencias y Artes de Barcelona XXIII, 21: 415-439.

Vilaseca, S. 1949: "Avance al estudio de la Cueva del Filador, de Margalef (provincia de Tarragona)". Archivo Español de Arqueología XXII: 347-361.

Villaverde, V. 2004: “Arte mueble paleolítico en el Mediterráneo occidental: contexto y diversidad regional". En P. Arias y R. Ontañón (ed.): La materia del lenguaje prehistórico. El arte mueble paleolítico de Cantabria en su contexto. Instituto Internacional de Investigaciones Prehistóricas de Cantabria. Santander: 67-84.

Villaverde, V. 2005: “Arte Paleolítico mediterráneo: de la Cueva de la Pileta a la Cova de les Meravelles". En M. Hernández y J. A. Sole (eds.): Arte Rupestre 
en la España Mediterránea. Instituto Alicantino de Cultura "Juan Gil-Albert". Alicante: 17-44.

Villaverde, V.; Martínez Valle, R.; Domingo, I.; López Motalvo, E. y García Robles, M. R. 2000: “Abric de Vicent: un nuevo abrigo con Arte Levantino en Millares (Valencia) y valoración de otros hallazgos en la zona". Pré-História Recente da Península Ibérica, Actas do $3{ }^{\circ}$ Congresso de Arqueologia Peninsular (Oporto, 2000) IV: 433-445. Porto.
Villaverde, V.; Cardona, J. y Martínez-Valle, R. 2009: "L'art pariétal de la grotte Les Meravelles. Vers une caractérisation de l'art paléolithique pré-magdalénien du versant méditerranéen de la Péninsule Ibérique". L'Anthropologie 113: 762-793.

Weninger, B.; Jöris, O. y Danzeglocke, U. 2007: CalPal-2007. Cologne Radiocarbon Calibration \& Palaeoclimate Research Package. http://www.CalPal.de/ (consulta 15-III-2010). 